\title{
Influencia de la edad en la calidad de los componentes parciales del sueño en la población general
}

\author{
G. de Teresa Romero, V.M. Vázquez García*, A.A. Álvarez Hurtado**, \\ J.L. Carretero Ares***, F. Alonso del Teso****, \\ R.M. GONZÁLEZ LAS HERAs***** \\ Residente de MFyC, C.S. Parquesol. *Especialista en MFyC, C.S. Tórtola. \\ **Residente de M FyC. C.S. Casa del Barco. ***Residente de M FyC. C.S. La Victoria. \\ ****Residente de Psiquiatría, Hospital Clínico Universitario. \\ ******Enfermera de Atención Primaria. Valladolid
}

\section{RESUMEN}

Objetivos: determinar cómo influye la edad en la calidad global del sueño y en sus componentes.

Diseño: estudio descriptivo transversal basado en encuesta personal y aplicación del Cuestionario del Sueño de Pittsburgh en 1.053 individuos selec cionados de entre todos los mayores de 18 años que acudieron a nuestros Centros de Salud durante el periodo de estudio (1/09/97 a 31/08/98). Test de $\chi^{2}$ para comparación de variables cualitativas, ANOVA para cuantitativas y prueba de Scheffé de contrastes a posteriori para comparaciones múltiples. Se asu mió un nivel de significación estadística de 0,05.

Resultados: el 33,5\% de los pacientes refirieron alteraciones del sueño (17,4\% leves, 9,8\% modera dos y 6,3\% severos). Las mujeres presentaron pun tuaciones significativamente peores que los hombres $(p<0,01)$. Tras los 54 años la calidad del sueño em peora progresivamente, no existiendo diferencias sig nificativas a partir de estas edades. Excepto en la disfunción diurna, en todos los demás componentes existen diferencias significativas a partir de los 54 años, presentando una mayor alteración en el com ponente de perturbaciones externas y menor en los de eficiencia habitual y uso de medicación hipnótica.

Conclusiones: existe una alta prevalencia de al teraciones de la calidad del sueño en nuestra pobla ción. Por encima de los 54 años no se está contento con la calidad de sueño, siendo esta disconformidad mayor entre las mujeres. Parece existir asociación entre la edad y el deterioro de la misma, siendo las perturbaciones extrínsecas el componente que más la deteriora. Se necesitan otro tipo de estudios para determinar la causa de estas perturbaciones.

Palabras clave: Calidad de sueño. Edad. Cuestio nario del sueño de Pittsburgh.
Influence of age in the quality of sleep partial components in the community

\section{ABSTRACT}

Objectives: to assess how age determines global sleep quality and its partial components.

Design: transversal descriptive study based on personal interview and the application of the Pitts burgh Sleep Quality Index in 1,053 persons selected among those over 18 years old who visited our Health Care Centers during the study period (01/09/97 to 31/08/98). $\chi^{2}$ test to compare qualitati ve variables, ANOVA for quantitative ones and Scheffé test for multiple comparisons. A p<0,05 statistical significance threshold was set.

Results: 33,5\% of the patients referred sleep pro blems (17,4\% mild, 9,8\% moderate and 6,3\% seve $r e)$. Women obtained significantly worse scores than men $(p<0,01)$. Over the age of 54 , sleep quality pro gressively worsened, without obtaining significant differences after this age. Except for daytime dys function, all the other sleep partial components sho wed significant differences over 54 years old, sho wing the highest scores for the external perturbations component and the lowest for the usual efficiency and hypnotic drug use components.

Interpretation: there is a high prevalence of sleep quality perturbations in our community. People over 54 are not happy with their sleep quality, especially women. There seems to be an association between age and sleep quality worsening, being external per turbations the component that influences the most. Some other type of studies are needed to establish the etiology of these alterations.

Key words: Sleep quality. Age. Pittsburgh Sleep Quality Index. 


\section{INTRODUCCIÓN}

Es un hecho contrastado en la literatura científica que la edad está en relación directa con el empeoramiento de la calidad del sueño, bien sea por las propias variaciones circadianas que se producen con el envejecimiento, por alteraciones primarias o por consecuencia directa de la mayor prevalencia de patologías asociadas y uso de fármacos que estos pacientes sufren $^{1-7}$. Se ha comprobado que la mala calidad de sueño es, en edades avanzadas, un importante factor de riesgo de morbimortalidad ${ }^{8}$. Además, es necesario tener en cuenta el número creciente de ancianos que reside en asilos y/o residencias, lo cual supone un factor de riesgo adicional para padecer alteraciones del sueño ${ }^{9,10}$. Las alteraciones del sueño en edades avanzadas son tan frecuentes que casi el $50 \%$ de los fármacos hipnóticos prescritos en Atención Primaria (AP) van dirigidos a la población de pacientes mayores de 65 años ${ }^{9}$. Sin embargo, no existen demasiados estudios centrados en determinar cómo se afecta el sueño no en su globalidad, sino en sus distintos componentes parciales. Éste es el objetivo de este trabajo, buscando un abordaje más efectivo y eficiente de esta patología cada vez más prevalente en sociedades como la nuestra ${ }^{6}$.

\section{MATERIAL Y MÉTODOS}

La población a estudio fue una muestra de 1.053 pacientes de entre los mayores de 18 años que acudieron a los Centros de Salud de nuestra población (359.027 habitantes) durante el periodo de estudio (01/09/97 a 31/08/98). Se trata de un estudio descriptivo transversal llevado a cabo mediante entrevista personal y aplicación del cuestionario de Pittsburgh corregido para evaluar la calidad del sueño de cada uno de los pacientes. El muestreo se realizó mediante diseño bietápico con unidades de primera (Centros de Salud) y segunda etapa (individuos). La muestra de Centros de Salud se extrajo utilizando muestreo estratificado en base al tamaño de la población atendida y su localización, resultando seleccionados cuatro Centros Urbanos y tres Rurales. La muestra dentro de cada Centro se seleccionó en base a muestras por cuotas de edad y sexo, intentando reproducir las proporciones de individuos existentes en el censo en ese momento. La entrevista personal se realizó por un entrevistador experto a cada paciente durante la primera visita dentro del periodo de estudio. Se utilizó el Cuestionario del Sueño de Pittsburgh (PSQI) creado por Buysse et $\mathrm{al}^{3,11}$ y corregido por Royuela et $\mathrm{al}^{2}$, para valorar la calidad de sueño del mes anterior a su aplicación. Consta de 24 preguntas, 19 de las cuales son contestadas por el propio sujeto y 5 por el compañero de habitación, siendo puntuados únicamente los 19 ítems autoaplicados. Éstos se combinan entre sí para formar siete componentes, cada uno de los cuales recibe una puntuación de 0 a 3 (de menor a mayor alteración del sueño), oscilando la puntuación global entre 0 y 21 puntos. La estructuración de estos siete componentes permite que los resultados del PSQI sean comparables con los que se obtienen de forma rutinaria en los pacientes que consultan a diario por esta patología. El punto de corte se establece en 5 puntos, considerándose "buenos dormidores" a aquéllos que obtienen una puntuación global menor o igual a $5^{3}$. Este cuestionario presenta una consistencia interna, medida mediante el coeficiente $\alpha$ de Cronbach, de 0,83 , una sensibilidad del $89,6 \%$ y una especificidad del $86,5 \%$. los mismos valores de consistencia interna, sensibilidad y especificidad. Los datos obtenidos se introdujeron en una base de datos del programa R-Sigma. Para su análisis se emplearon porcentajes y medias acompañados de sus respectivas desviaciones estándar. Se emplearon los test $\chi^{2}$ de Pearson para la comparación de variables cualitativas, ANOVA para variables cuantitativas y la prueba de Scheffé de contrastes a posteriori para los contrastes múltiples. En todo momento se asumió un nivel de significación estadística de 0,05 .

\section{RESULTADOS}

Eran varones $493(46,8 \%)$ y $560(53,2 \%)$ mujeres. La distribución de la población en cuanto a edad queda reflejada en la figura 1 . Los perfiles de sexo y edad de los pacientes fueron comparables a los de la población general. La puntuación total media de toda la muestra fue de $4,71^{3,59}$. El $66 \%$ obtuvo una puntuación total menor o igual de 5 y el 33,5\% mayor. De entre los considerados "malos dormidores", un $51,5 \%$ lo fueron en grado leve (6-8 puntos), un $29,4 \%$ moderado (9-11 puntos) y un $19,1 \%$ severo (>11 puntos). Del total de la población, el 17,4\% presentó trastornos leves (6-8 puntos), el 9,8\% moderados (9-11 puntos) y el 6,3\% graves (>11 puntos). La

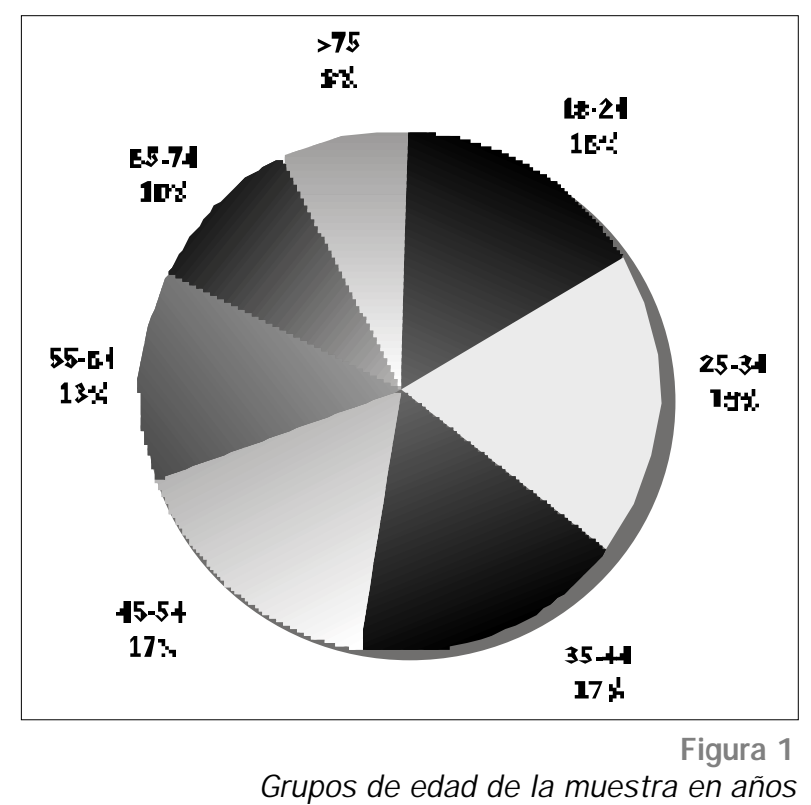


puntuación media total en los hombres fue de 4,33 3,4 y la de las mujeres $5,05^{3,72}(\mathrm{p}<0,01)$. Los resultados por grupos de edad de la puntuación total del PSQI quedan reflejados en la tabla $\mathrm{I}$, en la que vemos cómo, en términos generales, la puntuación va aumentando a la vez que lo hace la edad.

Los siete componentes en los que se divide el cuestionario son los siguientes:

-Calidad subjetiva de sueño: el 34,2\% de la población refirió tener muy buena calidad subjetiva de sueño, el $46,3 \%$ buena, el $16,8 \%$ mala y el $2,7 \%$ muy mala. Globalmente, el $80,5 \%$ de nuestra población está satisfecha con su calidad de sueño. Las mujeres presentaron peores resultados en este componente $[0,95(0,77)$ vs $0,79(0,77)](\mathrm{p}<0,01)$. Existieron diferencias significativas entre los menores de 55 años y los de 55 o más. A partir de los 54 años la calidad subjetiva empeoró progresivamente, aunque sin existir diferencias significativas entre ellos.

- Latencia de sueño (suma del tiempo que el sujeto cree que tarda en dormirse y del número de veces que el sujeto no ha podido coger el sueño en la primera media hora en el último mes): el $40 \%$ de la población presentó 0 puntos, el $31 \% 1$ punto, el $15,6 \% 2$ puntos y el 12,4\% 3 puntos. La puntuación media en este componente fue de 0,99 (1,03). La mayoría de los encuestados presentó una latencia aceptable (<30 minutos). Las mujeres presentaron peores resultados $\left[\begin{array}{lllll}1,07 & (1,06) & \text { vs } & 0,9 & (0,98)\end{array}\right]$ $(\mathrm{p}<0,05)$. Existieron diferencias significativas entre los menores de 55 años y los de 55 o más. A partir de los 54 años la latencia del sueño empeoró progresivamente, no existiendo diferencias significativas entre ellos.

-Duración del sueño (número de horas que el sujeto cree haber dormido): el 68,2\% refirió dormir más de 7 horas, el 21,4\% entre 6-7 horas, el 7\% entre 5-6 horas y el 3,4\% menos de 5 horas. La puntuación media fue $0,46(0,77)$. No existieron diferencias en cuanto a sexo. Existieron diferencias significativas entre los menores de 55 años y los de 55 o más. A partir de los 54 años la duración del sueño empeoró progresivamente, no existiendo diferencias significativas entre ellos.

Tabla I

RESULTADOS GLOBALES DEL CUESTIONARIO DE PITTSBURGH POR GRUPOS DE EDAD

EDAD (AÑOS) PUNTUACIÓN: MEDIA (DS)

$18-24$

$25-34$

$35-44$

$45-54$

$55-64$

$65-74$

$>74$

Total Media
$3,72(2,75)$

$3,91(2,72)$

$3,71(3,18)$

$4,77(3,67)$

$5,67(3,99)$

$6,51(4,21)$

$6,79(4,18)$
-Eficiencia habitual del sueño (cociente entre el tiempo que el sujeto cree dormir y el que declara que permanece acostado): el $76,4 \%$ obtuvo una puntuación de 0 , el $11,8 \% 1$ punto, el 7,9\% 2 puntos y el $3,9 \% 3$ puntos. La puntuación media fue de 0,39 $(0,79)$. No hubo diferencias en cuanto a sexo. Sí las hubo entre el grupo de menos de 45 y el de 55-64 años y entre los menores de 55 y los mayores de 64 .

- Perturbaciones extrínsecas del sueño (incluyen los despertares nocturnos, las alteraciones miccionales, los episodios de tos, los problemas respiratorios, los ronquidos, la sensación distérmica, las pesadillas y los dolores): un 13,3\% manifestó no haber sufrido perturbación alguna, el 69,4\% refirió perturbaciones leves, un $16,7 \%$ moderadas y un $0,6 \%$ severas. La puntuación media fue de $1,05(0,57)$. No hubo diferencias entre sexos. Existieron diferencias significativas entre el grupo de menos de 45 y el de $45-74$ años y entre el de menos de 55 y el de más de 75 años.

-Uso de medicación hipnótica en el último mes: el $83 \%$ refirió no haber consumido ningún fármaco, el $6,1 \%$ menos de uno a la semana, el $3,2 \%$ de 1 a 2 por semana y el 7,7\% más de 3 por semana. La puntuación media fue de $0,36(0,87)$. Las mujeres refirieron mayor consumo que los hombres $[0,46(0,97)$ vs $0,23(0,71)](\mathrm{p}<0,01)$. Existieron diferencias significativas entre el grupo de menos de 35 y el de $45-54$ años y entre el de menos de 45 y el de más de 54 .

-Disfunción diurna (suma de la presencia o ausencia de somnolencia diurna y la existencia o no de desgana en las actividades diurnas): el 57,5\% de la población no presentó disfunción diurna (0 puntos), el $28,3 \%$ leve ( 1 punto), el $12,3 \%$ moderada ( 2 puntos) y el $1,9 \%$ severa (3 puntos). La puntuación media fue de $0,59(0,78)$. No se encontraron diferencias significativas en cuanto a sexo y/o edad. La puntuación de cada uno de estos componentes según la distribución por edades de la población de estudio queda reflejada en la tabla II.

\section{DISCUSIÓN}

La puntuación total media de nuestra población refleja que ésta tiene una calidad de sueño aceptable. Nuestros resultados indican un empeoramiento de la calidad de sueño a medida que aumenta la edad, coincidiendo con los de Dement et $\mathrm{al}^{12}$. El grupo de edad que manifestó disfrutar de mayor calidad de sueño fue el de 35 a 44 años. Los sujetos que obtuvieron puntuaciones superiores a 5 ("malos dormidores") fueron los mayores de 55 años. A excepción del componente de disfunción diurna, en el que no se observaron diferencias significativas, en el resto de componentes (C1-C6) existieron diferencias significativas a partir de los 54 años. La calidad subjetiva del sueño (C1) empeoró notablemente a partir de los 54 años. A partir de esta edad los pacientes refirieron tardar más en conciliar el sueño, dormir menos tiempo, despertarse más frecuentemente y tener un sueño 
Tabla II

\begin{tabular}{|c|c|c|c|c|c|c|c|}
\hline \multicolumn{8}{|c|}{ PUNTUACIÓN DE LOS COMPONENTES PARCIALES DEL SUEÑO SEGÚN LA EDAD } \\
\hline EDAD & $\begin{array}{l}\text { Calidad } \\
\text { Subjetiva }\end{array}$ & Latencia & Duración & $\begin{array}{c}\text { Eficiencia } \\
\text { Habitual }\end{array}$ & $\begin{array}{c}\text { Perturbaciones } \\
\text { extrínsecas }\end{array}$ & $\begin{array}{l}\text { Medicación } \\
\text { hipnótica }\end{array}$ & $\begin{array}{l}\text { Disfunción } \\
\text { diurna }\end{array}$ \\
\hline $18-24$ & $0,77(0,79)$ & $0,89(0,95)$ & $0,27(0,54)$ & $0,13(0,45)$ & $0,85(0,48)$ & $0,07(0,29)$ & $0,74(0,78)$ \\
\hline $25-34$ & $0,74(0,70)$ & $0,93(0,98)$ & $0,31(0,57)$ & $0,21(0,50)$ & $0,88(0,51)$ & $0,13(0,38)$ & $0,71(0,76)$ \\
\hline $35-44$ & $0,74(0,79)$ & $0,72(0,90)$ & $0,33(0,67)$ & $0,22(0,56)$ & $0,90(0,52)$ & $0,20(0,64)$ & $0,54(0,75)$ \\
\hline $45-54$ & $0,90(0,74)$ & $0,97(1,01)$ & $0,43(0,67)$ & $0,30(0,66)$ & $1,14(0,58)^{1-3}$ & $0,51(1,02)^{1-2}$ & $0,55(0,81)$ \\
\hline $55-64$ & $1,10(0,85)^{1-3}$ & $1,35(1,20)^{1-3}$ & $0,70(0,91)^{1-3}$ & $0,55(0,94)^{1-3}$ & $1,18(0,53)^{1-3}$ & $0,55(1,09)^{1-3}$ & $0,46(0,68)$ \\
\hline $65-74$ & $1,13(0,80)^{1-3}$ & $1,37(1,21)^{1-3}$ & $0,65(1,03)^{1-3}$ & $0,90(1,08)^{1-5}$ & $1,40(0,52)^{1-3}$ & $0,78(1,25)^{1-3}$ & $0,42(0,78)$ \\
\hline$>75$ & $1,14(0,63)^{1-3}$ & $1,38(1,19)^{1-3}$ & $0,72(1,13)^{1-3}$ & $1,09(1,24)^{1.5}$ & $1,27(0,55)^{1-4}$ & $0,66(1,15)^{1-3}$ & $0,57(0,86)$ \\
\hline TOTAL & $0,88(0,78)$ & $0,99(1,03)$ & $0,46(0,77)$ & $0,39(0,79)$ & $1,05(0,57)$ & $0,36(0,87)$ & $0,59(0,78)$ \\
\hline
\end{tabular}

En números superíndice se indican los grupos entre los que existen diferencias estadísticamente significativas $(p<0,05)$.

menos eficiente, encontrándose diferencias estadísticamente significativas $(\mathrm{p}<0,05)$ con los sujetos más jóvenes, resultados que coinciden con los ya publicados por algunos autores ${ }^{12}$. La eficiencia habitual (C4) fue significativamente menor entre los mayores de 65 años, lo cual puede ser explicado a partir de las variaciones circadianas fisiológicas, con lo cual se tendería a una fragmentación del sueño y a la aparición de hipersomnia diurna ${ }^{13,14}$. Aunque con un inicio tal vez más temprano del que pudiera esperarse, las perturbaciones extrínsecas (C5) se hicieron significativamente más frecuentes a partir de los 54 años, coincidiendo probablemente con la mayor prevalencia de pluripatología que a estas edades se sufre. Cabe destacar el bajo porcentaje de individuos que refirió polimedicación $(10,9 \%)$, así como el hecho de que los mayores de 74 años consumieran menos fármacos que los de 65 a 74, si bien las diferencias no fueron significativas. El hecho de que la disfunción diurna (C7) se vea más afectada en los jóvenes lo relacionan algunos autores ${ }^{15,16}$ con que esta población duerme menos tiempo del fisiológicamente necesitado, mientras que los ancianos pasarían más tiempo acostados del fisiológicamente necesitado, lo que explicaría que en ellos la eficiencia del sueño se vea más afectada que la disfunción diurna. Estos resultados discrepan con los publicados por Roehrs et $\mathrm{al}^{17}$, quienes encontraron igual afectación en la disfunción diurna en la población joven que en la de mayores de 55 años. En nuestro estudio, los componentes que más afectados se vieron fueron la calidad subjetiva, la latencia del sueño y las perturbaciones extrínsecas. El perfil del grupo de nuestro trabajo presentó un pico máximo en el C5 (perturbaciones extrínsecas) y dos valles en los C4 (eficiencia habitual) y C6 (uso de medicación hipnótica). Al igual que en otros estu$\operatorname{dios}^{8}$, los ancianos de nuestra comunidad no están satisfechos con su calidad de sueño, siendo ésta peor en las mujeres que en los hombres.

Una limitación de este estudio podría ser el que esté basado en datos subjetivos (respuestas de los pacientes), si bien los datos siguen siendo de interés, pues es la experiencia subjetiva la que hace a los mismos acudir a nuestra consulta. En la patología del sueño, como en tantas otras, el éxito del diagnóstico dependerá del reconocimiento precoz del problema, de la valoración de la magnitud de su impacto en la vida del paciente y de su correcta evaluación. El PSQI se presenta como un instrumento fiable, válido y aplicable en la consulta diaria de Atención Primaria para la valoración clínica del insomnio en el estado inicial del cribado de casos. Además, no requiere la especialización por parte del examinador que puede requerir una evaluación más detallada, por lo que puede ser especialmente útil para los Equipos de Atención Primaria. Su principal ventaja es que al analizar específicamente cada componente del sueño nos permite:

-Obtener información sobre la calidad subjetiva del sueño (cómo concibe el propio paciente su problema).

- Clasificar el tipo de insomnio padecido (predormicional, intradormicional o postdormicional).

- Detectar problemas distintos al propio funcionamiento intrínseco del sueño que pueden afectar a éste (alteraciones respiratorias, cardiocirculatorias, miccionales, neurológicas, psiquiátricas, ingesta de medicamentos, etc.). Este reconocimiento y actuación precoz adquiere especial importancia en los ancianos, en los que se ha demostrado que la mala 
calidad de sueño es un importante factor de riesgo de mortalidad $^{8}$, asociándose en residencias y asilos de forma más significativa al riesgo de muerte e ingresos hospitalarios que la edad, la dificultad para la realización de actividades diurnas, la escasa salud física y/o el bajo nivel socioeconómico como factores independientes ${ }^{18}$. No todas las alteraciones del sueño del anciano son benignas y secundarias a una desincronización fisiológica secundaria al envejecimiento ${ }^{19}$. Muchas veces existe algún factor etiológico primario o secundario que justifica la realización de un estudio complementario adecuado ${ }^{5,20,21}$. Para obtener un diagnóstico efectivo es necesario emplear todos los medios disponibles a nuestro alcance (revisión de la higiene del sueño, uso de medicamentos, información de la pareja, registros del sueño, control en casa, polisomnografía y/o valoración del impacto del problema en las actividades de la vida diaria del paciente) ${ }^{12}$. Hay que seguir insistiendo en la necesidad de incluir en la atención de estos pacientes una correcta anamnesis dirigida a enfocar adecuadamente el diagnóstico de las alteraciones del sueño, así como en conocer las bases de un estudio correcto y las indicaciones de remisión a una unidad especializada antes de iniciar la farmacoterapia ${ }^{22}$. El médico de AP se encuentra en una situación ideal para identificar este problema y abordarlo precozmente, por lo que es necesario resaltar la importancia de la adquisición de una formación adecuada en los principios básicos de la medicina del sueño y sus avances recientes ${ }^{10,23}$, así como mantener una relación bidireccional con el resto de profesionales que trabajan en este campo (otros médicos de familia, geriatras, psicogeriatras, neumólogos y electrofisiólogos). Tal vez debiera considerarse la introducción de estos temas en la formación de pregrado y la oferta de una más completa formación continuada de postgrado para mejorar la situación actual.

\section{CORRESPONDENCIA:}

G. de Teresa Romero

C.S. Parquesol

C/ Ciudad de la Habana 17

47014 Valladolid

\section{Bibliografía}

1. Foley DJ, Monjan AA, Brown SL, Simnonsick EM, Wallace RB, Blazer GG. Sleep complaints among eldely persons: an epidemiologic study of three comunities. Sleep 1995; 18 (6): 425-32.

2. Royuela Rico A, Macías Fernández JA. Propiedades clinimétricas de la versión castellana del cuestionario de Pittsburgh. Vigilia-Sueño 1997; 9 (2): 81-94.

3. Buysse DJ, Reynolds CF, Monk TH. Quantification of subjective sleep quality in healthy elderly men and women using the Pittsburgh Sleep Quality Index (PSQI). Sleep 1991; 12: 529-36.

4. Gislason T, Almquist M, Kristbjarnarson H, Benediktsdottir B. Sleep habits and sleep disturbances among the elderly: an epidemiological survey. Journal os Internal Medecin 1993; 234 (1): 31-9.

5. Bundlie SR. Sleep in aging. Geriatrics 1998 Sep; 53 (Suppl 1): S41-3.

6. Cadieux RJ, Adams DG. Sleep disorders in older patients. Conservative treatment is usually enough. Postgrad Med 1992; 91 (4): 403-4, 407-10, 415-6.

7. Daly MP. Sleep disorders in the elderly. Prim Care 1989;16 (2): 475-88.

8. Kripke DF, Simons EN, Garfinkel L, Hammond EC. Short and long sleep and sleeping pills: Is increased mortality associated?. Arch Gen Psychiatry 1979; 36: 103-16.

9. Gottlieb GL. Sleep disorders and their management. Special considerations in the elderly. Am J Med 1990; 88: 29S-33S.

10. Martin J, Shoat T, Gehrman PR, Ancoli-Israel S. Sleep in the elderly. Respir Care Clin N Am 1999; 5 (3): 461-72.

11. Buysse DJ, Reynolds CF, Monk TH, Berman SR, Kupffer DJ. The Pittsbuegh sleep quality index: A new instrument for psychiatric practice and research. Psychiatry Research 1989; 28 : 193-213.

12. Dement WC, Miles LE, Carskadon MA. "White paper" on sleep and aging. Am Geriat Soc 1982; 30: 25-50.
13. Campbell SS, Gillin JK, Kripke DF, Erikson P, Clopton P. Gender differences in the circadian temperature rhythms of healthy elderly subjects: Relationships to sleep quality. Sleep 1989; 12: 529-36.

14. Hoch CC, Reynolds CF, Kupfer DJ, Berman SR. Stability of EEG sleep and sleep quality in healthy seniors. Sleep 1988; 11 : 521-27.

15. Susman JL. Sleep in the elderly: A practical approach. J Fam Physician 1989; 29 (5): 528-33.

16. Lecendreaux M. Troubles du sommeil chez l'enfant et l'adolescent. Encycl Méd Chir. Psychiatrie 1996; 37-200-e-38. Pédiatrie 1996; 4-102-A-10.

17. Roehrs T, Zorick F, Sicklesteel J, Witting R, Roth T. Age-related sleep-wake disorders at a sleep disorder center. Jam Geriatr Soc 1983 Jun; 31 (6): 364-70.

18. Pollack CP, Perlick D, Linsner JP, Wenston J, Hsieh F. Sleep problems in the community elderly as predictors of death and nursing home placement. J Community Health 1990; 15: 123-35.

19. Haponik EF, Frye AW, Richards B, Wymer A, Hinds A, Pearce $\mathrm{K}$, et al. Sleep history is neglected diagnostic information. Challenges for primary care physicians. J Gen Intern Med 1996; 11 (12): 759-61.

20. Neubauer DN. Sleep problems in the elderly. Am Fam Physician 1999; 59(9): 2551-8, 2559-60.

21. Morgan K, HealeyDW, Healey PJ. Factors influencing persistent subjective insomnia in the old age: A folluw-up study of good and poor sleepers aged 65 to 74. Age Ageing 1989; 18: 177.

22. Becker PM, Jamieson AO. Common sleep disorders in the elderly: diagnosis and treatment. Geriatrics 1992 Mar; 47 (3): 41-2, 45-8, 51-2.

23. Ancoli-Israel S. Epidemiology of sleep disorders. Clin Geriatr Med 1989; 5 (2): 247-62. 